\title{
$541996-17910$ \\ HIGH GAIN GaAs \\ CONF- $960685-3$ \\ PHOTOCONDUCTIVE SEMICONDUCTOR SWITCHES \\ FOR GROUND PENETRATING RADAR
}

\author{
High Power Electromagnetics Department, MS 1153 \\ Sandia National Laboratories \\ Albuquerque, NM 87185-1153 \\ Tel. (505) 845-7096 \\ D. J. Brown \\ Ktech Corporation \\ Albuquerque, NM 87110
}

G. M. Loubriel, J. F. Aurand, M. T. Buttram, F. J. Zutavern, W. D. Helgeson, and M. W. O'Malley

\begin{abstract}
The ability of high gain GaAs Photoconductive Semiconductor switches (PCSS) to deliver high peak power, fast risetime pulses when triggered with small laser diode arrays makes them suitable for their use in radars that rely on fast impulses. This type of direct time domain radar is uniquely suited for observation of large structures under ground because it can operate at low frequencies and at high average power. This paper will summarize the state-of-the-art in high gain GaAs switches and discuss their use in a radar transmitter. We will also present a summary of an analysis of the effectiveness of different pulser geometries that result in transmitted pulses with varying frequency content. To this end we developed a simple model that includes transmit and receive antenna response, attenuation and dispersion of the electromagnetic impulses by the soil, and target cross sections.
\end{abstract}

\section{Introduction on High Gain GaAs Switches}

This research has focused on optically triggered, high gain GaAs switches for impulse sources for ultrawide bandwidth (UWB) transmitters. The practical significance of this high gain switching mode is that the switches can be activated with very low energy optical triggers, allowing for compact sources with very small jitter. ${ }^{1}$ The GaAs switches used in this experiment are lateral switches: they have two contacts on one side of a wafer separated by an insulating region of intrinsic material. At electric fields below $4 \mathrm{kV} / \mathrm{cm}$, the $\mathrm{GaAs}$ switches are activated by the creation of, at most, one electron hole pair per photon absorbed. This linear mode demands high laser power, and after the light is extinguished, the carrier density decays in 1-10 ns. At higher electric fields the field induces carrier * This work supported by DOE contract DE-AC0494AL85000. multiplication so that the amount of light required is reduced by as much as five orders of magnitude. ${ }^{1,2}$ This high gain mode is characterized by fast current rise times ( $200 \mathrm{ps})$. In the "on" state of the high gain switch there is a characteristic, constant field across the switch called the lock-on field. The switch current is circuit-limited provided the circuit maintains the lockon field. As the initial voltage increases, the switch risetime decreases and the trigger energy is reduced. The PCSS are being tested for use in applications such as: UWB transmitters, firing sets, electro-optic modulators, current interrupters, and pulsed power applications such as MV accelerators. Each of these applications imposes a different set of requirements on switch properties. Table 1 shows our best results obtained with the switches for these applications.

Table 1. Results of tests with high gain GaAs switches. The first column is the best results obtained in various, independent tests. The second column are the results from a single system.

\begin{tabular}{|l|c|c|}
\hline \multicolumn{1}{|c|}{$\begin{array}{c}\text { Table I } \\
\text { Parameter }\end{array}$} & $\begin{array}{c}\text { Best } \\
\text { Results* }\end{array}$ & $\begin{array}{c}\text { Simultaneous } \\
\text { Results }\end{array}$ \\
\hline Switch Voltage (kV) & 155 & 100 \\
\hline Switch Current (kA) & 7.0 & 1.26 \\
\hline Peak Power (MW) & 120 & 48 \\
\hline Rise time (ps) & 430 & 430 \\
\hline R-M-S jitter (ps) & 150 & 150 \\
\hline Trigger Energy (nJ) & 13 & 180 \\
\hline Optical Trigger Gain & $10^{5}$ & $10^{5}$ \\
\hline Repetition Rate (Hz) & 1,000 & 1,000 \\
\hline Electric Field (kV/cm) & 100 & 67 \\
\hline $\begin{array}{l}\text { Device Lifetime } \\
\text { (\# pulses) }\end{array}$ & $6 \times 10^{6}$ & $\begin{array}{c}5 \times 10^{4}, \\
\text { (at } 77 \mathrm{kV})\end{array}$ \\
\hline
\end{tabular}

* Not all the results are simultaneous. 
DISCLAIMER

Portions of this document may be illegible in electronic image products. Images are produced from the best available original document. 


\section{Ground Penetrating Radar}

The system we are building seeks to increase penetration depth over conventional systems by using: 1) high peak power, high repetition rate operation that gives high average power, 2) low center frequencies that better penetrate the ground, and 3) short duration impulses that allow for the use of downward looking, low flying platforms that increase the power on target relative to a high flying platform. Specifically, chirped pulses that are a microsecond in duration require (it is difficult to receive during transmit) platforms above $150 \mathrm{~m}$ (and typically $1 \mathrm{~km}$ ) while this system, theoretically could be at $10 \mathrm{~m}$ above the ground. The power on target decays with distance squared so the ability to use low flying platforms is crucial to high penetration. Clutter is minimized by time gating the surface clutter return. Short impulses also allow gating (out) the coupling of the transmit and receive antennas.

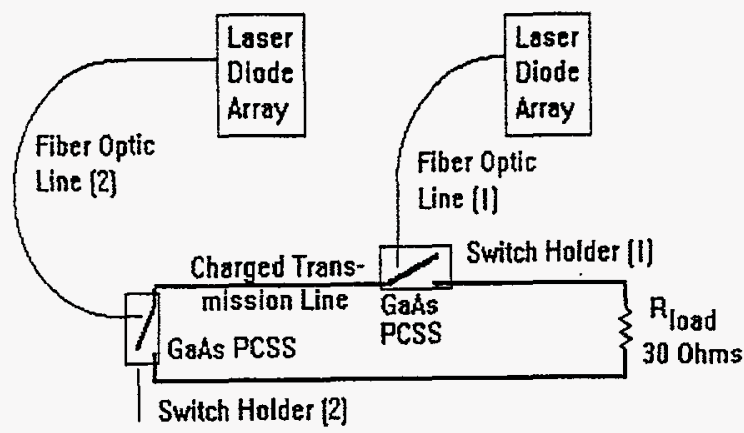

Figure 1. A short (1 ns), $47 \Omega$ transmission line (the charge line) was charged to high voltage at a burst repetition rate of $1 \mathrm{kHz}$. Two switches were used on either side of the line to discharge the line into a $30 \Omega$ load.

The feasibility of using GaAs switches to create voltage pulses suitable for driving UWB antennas has been previously demonstrated. ${ }^{3}$ In that study we charged a nominally $1.0 \mathrm{~ns}$ long, $47 \Omega$, parallel plate transmission line to voltages of about $100 \mathrm{kV}$. This line was discharged with either one or two switches into a $30 \Omega$ load (see Figure 1). The voltage on the line rose to a peak value ( $100 \mathrm{kV}$ in most cases, on occasion 110 $\mathrm{kV}$ ) with a risetime of $210 \mathrm{~ns}$. At peak voltage the laser diode arrays activated the switch and the line voltage dropped. The laser diode arrays (with most of their electronics) are about 2" by 2" in size and triggered the switches with as little as $90 \mathrm{~nJ}$ of energy.

If only one switch was triggered, the resulting load voltage was a unipolar pulse (of positive or negative polarity depending on which switch was triggered The highest current obtained was $1.26 \mathrm{kA}$ with a rise time of $430 \mathrm{ps}$ and a pulse width of $1.4 \mathrm{~ns}$. The peak power is $48 \mathrm{MW}$. This system operated in bursts of up to 5 pulses at a repetition rate of $1 \mathrm{kHz}$.

The second set of tests used both laser diodes, one per switch, to produce a bipolar pulse. In theory, with ideal switching, the bipolar pulse should be composed of two unipolar pulses of opposite polarity each with half the pulse width. Thus, we expect a bipolar pulse composed of a negative and a positive pulse, each with a width of $0.9 \mathrm{~ns}$. What we observe is a width of $1.0 \mathrm{~ns}$ for the negative pulse and $1.3 \mathrm{~ns}$ for the positive pulse. The reason for this is a timing error of about 200 ps. The minimum width should occur when both switches are triggered simultaneously. It is very important to trigger both switches at the same time to obtain full voltage and to obtain the proper waveform. In these tests, the switch jitter did not allow us to always reproduce the bipolar pulse. Although the bipolar waveform is harder to produce, it is more efficiently radiated out of an antenna. To reduce jitter it will be necessary to increase the laser energy. Fortunately, the laser diode array can be run, with electronics of the same size, at up to $1 \mu \mathrm{J}$ of energy.

\section{Model of a Ground penetrating Radar}

To analyze the relative merits of different waveforms (unipolar versus bipolar) with varying frequency content and to estimate the peak powers required for a given soil penetration, we have developed a simple radar model. This model ${ }^{4}$ includes transmit and receive antenna response, attenuation and dispersion of the electromagnetic impulses by the soil, and target cross sections. The target that we will choose is a metallic, square plate of size L buried at a depth $D$. To examine the radar equation for a buried object, we use the simple radar equation for the frequency dependent amplitude of the transfer function [radar-to-target-to-receiver]. The beam is assumed to be normal to the ground which is flat and featureless. Figure 2 shows two different voltage waveforms (input to antenna) that we will discuss in the paper: a unipolar pulse and a bipolar pulse. The formulas used to calculate these waveforms are either a Gaussian (sigma $=0.8 \mathrm{~ns}$ ) centered at $5 \mathrm{~ns}$ or a Gaussian times (t- $5 \mathrm{~ns}$ ). Both were normalized to peak amplitude of 1 (as shown). These waveforms were meant to mimic the results obtained with the previous impulse system.

The frequency spectrum of both of these waveforms is what determines their penetration characteristics. The unipolar pulse, due to its non zero integral, has peak frequency content at $0 \mathrm{MHz}(\mathrm{dc})$. 
Most of its energy content is from $0 \mathrm{MHz}$ to about 200 MHz. The bipolar pulse, on the other hand, has no frequency content at 0 and peaks at $200 \mathrm{MHz}$. Most of its energy content is from $100 \mathrm{MHz}$ to $350 \mathrm{MHz}$.
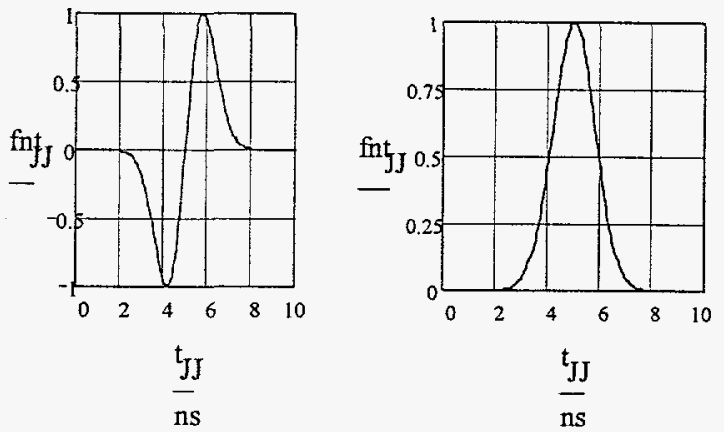

Figure 2. Voltage waveforms used in this study. On the left is a bipolar pulse, on the right is a unipolar pulse.

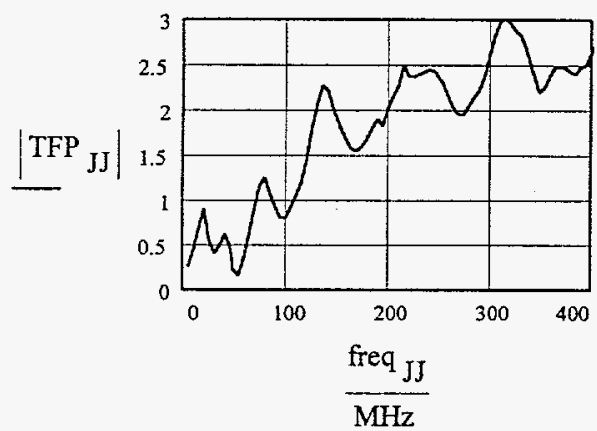

Figure 3. Antenna transfer function.

An existing antenna which may be used for this work is a large TEM horn with flared aperture plates and a high-voltage inline coaxial 'zipper' balun as the input section. It has a transfer function as shown in figure 3. This was developed by measuring two time domain waveforms. One was a bipolar voltage pulse created by a custom pulse generator with a $+/-100 \mathrm{~V}$ amplitude, and primary spectral content from 50 to 400 MHz. The other was the main radiated E-field, at a range of $8.0 \mathrm{~m}$. The transfer function was then formed by the complex ratio of the discrete Fourier transform of the radiated field divided by the transform of the input excitation voltage. The cross section (in Air) of the target (a metal plate $10 \mathrm{~m}$ by $10 \mathrm{~m}$ ) also increases with frequency. Because the antenna transfer function and the target cross section are larger at higher frequencies, the unipolar pulse will have less efficiency than the bipolar pulse. It is tempting to use waveforms that have very high frequency components. On the other hand, the attenuation by the soil is larger at higher frequencies. Figure 4 shows attenuation for San Antonio Clay Loam with a water content of $5 \%$. A similar calculation for sand shows little attenuation (the scale in the figure would range from -1.2 to 0 ).

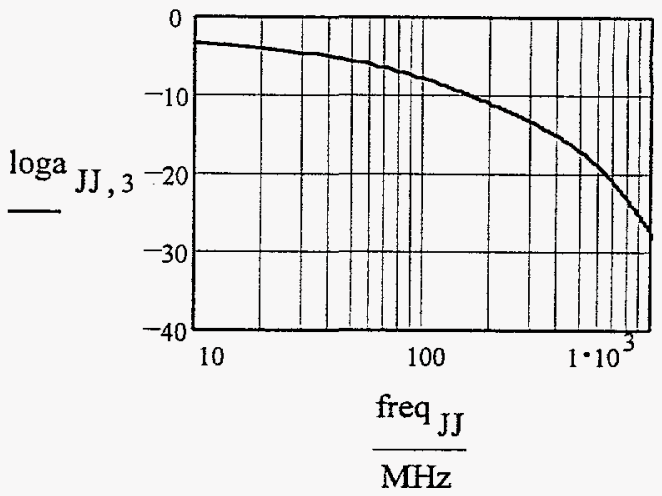

Figure 4. The $\log$ of the attenuation versus frequency for a penetration depth of $10 \mathrm{~m}$ for San Antonio clay loam, $5 \%$ water.

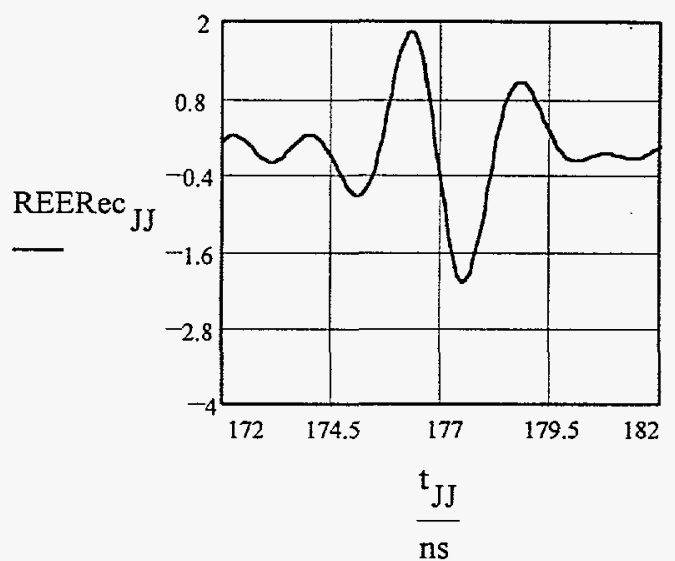

Figure 5. The electric field at the transmit site after transmission through sand for the bipolar pulse.

Using this information we can predict the receive waveform. Figures 5 and 6 show different receive waveforms for the bipolar voltage pulse. Figure 5 shows the return from penetration through $10 \mathrm{~m}$ of air and $10 \mathrm{~m}$ of soil. Note that the waveforms are similar to the derivative of the initial voltage pulse. Figure 6 shows the same type of results but with San Antonio clay loam with $5 \%$ water content $(4 \mathrm{~m}$ of air, $10 \mathrm{~m}$ of soil). The receive functions show two major effects. First, a large attenuation of about $10^{5}$ in intensity. Second, a great degree of what looks like dispersion in that the temporal extent of the pulse is now about $15 \mathrm{~ns}$. This last effect is mainly due to the high attenuation of 
the high frequencies that only allow the low (slow) frequencies to be received.

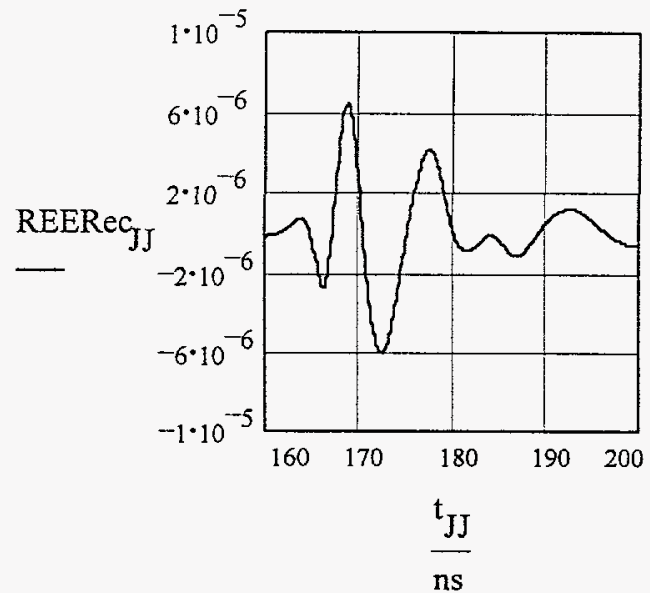

Figure 6. The electric field at the transmit site after transmission through San Antonio clay loam, 5\% water.

\section{Radar Transmitter}

Based on the above data, we are constructing a high peak power, high average power transmitter. The pulser section is similar to that in figure 1 but has a longer distance between the switches $(36.4 \mathrm{~cm})$ and its modulator is all solid state. Figure 7 shows a schematic of the modulator. Figure 8 shows the voltage output from the source to a $50 \Omega$ load.

\section{Conclusion}

These studies have shown that it is possible to obtain high peak power ( $48 \mathrm{MW}$ ) impulses in a system with an impedance of $30-50 \Omega$ using laser diode triggered PCSS operated in the high gain mode and that these pulses will penetrate soil with modest water content for depths of up to $10 \mathrm{~m}$.

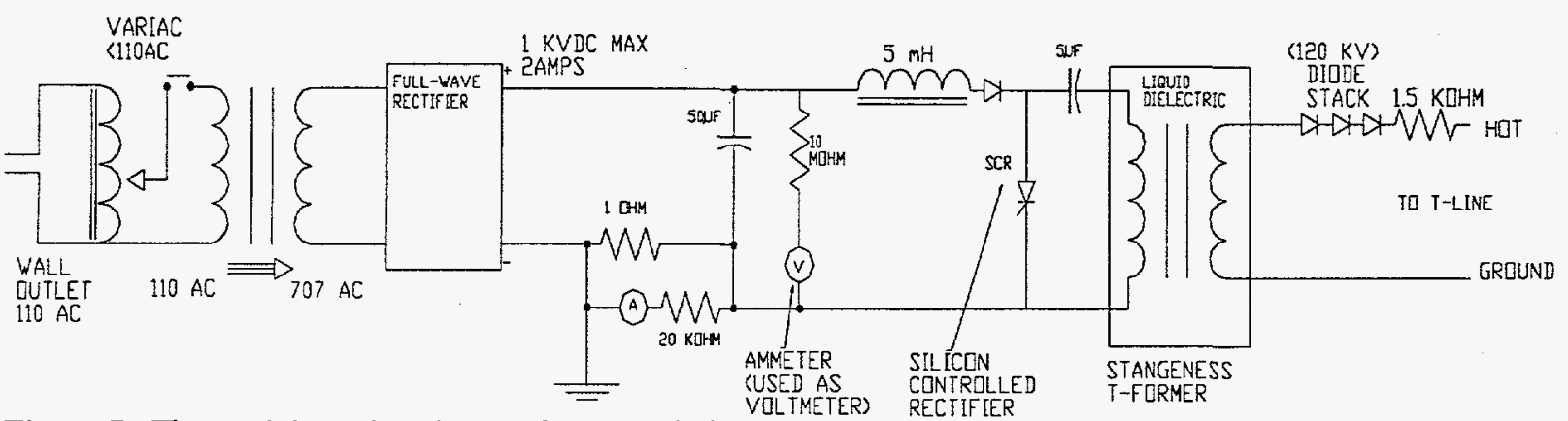

Figure 7. The modulator that charges the transmission (charge) line.

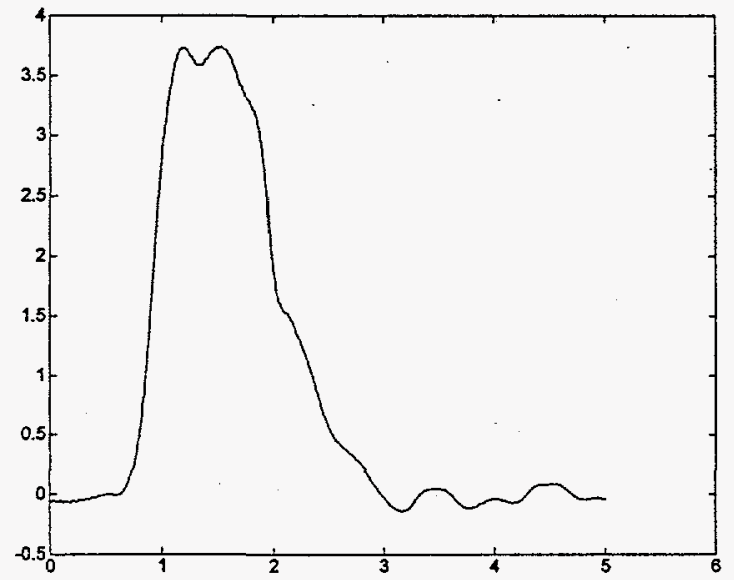

Figure 8. The voltage on the load for a unipolar pulse at $60 \mathrm{kV}$ charge. Peak voltage is $21.7 \mathrm{kV}$, risetime is $1.3 \mathrm{~ns}$, and the pulse width is $4.8 \mathrm{~ns}$, which results in $175 \mathrm{MHz}$ for the bipolar pulse.

\section{References}

1 G. M. Loubriel, et al, "Toward Pulsed Power Uses for Photoconductive Semiconductor Switches: Closing Switches," Proc. 6th IEEE Pulsed Power Conference, P. J. Turchi and B. H. Bernstein, eds., Arlington, VA, 1987, p. 145.

2 F. J. Zutavern, et al, "High Voltage Lateral Switches from $\mathrm{Si}$ or GaAs," in High-Power Optically Activated Solid-State Switches, A. Rosen and F. J. Zutavern, Eds., Artech House, Boston, 1993, p. 245.

3 G. M. Loubriel, et al, "High Gain GaAs Photoconductive Semiconductor Switches for Impulse Sources," Proc. of SPIE Optically Activated Switching Conference IV, SPIE Vol. 2343, p. 180, W. R. Donaldson, ed., Boston, MA, 1994.

4 G. M. Loubriel, et al, "Ground Penetrating Radar Enabled by High Gain GaAs Photoconductive Semiconductor Switches," in Ultra-Wideband. Short Pulse Electromagnetics 3, A. Stone, C. Baum, and L. Carin, eds., Plenum Press, NY, 1996. 